\title{
Structural effects in the production of neutrons, gammas and anti-neutrinos in fission
}

\author{
K.-H. Schmidt ${ }^{1, *}, M$. Estienne ${ }^{1}, M$. Fallot $^{2}, B . \mathrm{Jurado}^{3}$, and $C . \mathrm{Schmitt}^{4}$ \\ ${ }^{1}$ Rheinstr. 4, 64390 Erzhausen, Germany \\ ${ }^{2}$ Subatech CNRS/IN2P3-Université de Nantes-IMTA, 4 rue Alfred Kastler, 44307 Nantes, France \\ ${ }^{3}$ CENBG CNRS/IN2P3, Chemin du Solarium, B.P. 120, 33175 Gradignan, France \\ ${ }^{4}$ IPHC CNRS/IN2P3, 23 rue du Loess, B.P. 28, 67037 Strasbourg, France
}

\begin{abstract}
Structural effects in the production of light particles in fission are investigated. Most of these effects can be traced back to pairing correlations and shell effects and their dependencies on the composition of the fissioning system and its excitation energy. It is shown that the GEF code is able to reproduce most of these features and to explain their origin on the basis of established properties of nuclear matter as well as concepts and laws of general validity. Predictions for systems with scarce or no experimental information can also be made.
\end{abstract}

\section{Introduction}

Light particles can be emitted during all processes connected to nuclear fission. It begins with the emission of nucleons, mostly neutrons, (before and after formation of a compound nucleus) from the excited system in the first minimum. This process is connected with multichance fission, and it causes discontinuities in almost all fission observables as a function of the initial excitation energy. Further on, neutrons can be emitted in the fission process before reaching the scission point. Eventually, scission neutrons (in analogy to light charged particles in ternary fission) may be emitted. After neck rupture, the fragments normally evaporate several neutrons, partly during the acceleration phase. Most gammas (statistical and non-statistical) are emitted from the fragments, predominantly from states below the angular-momentum-dependent neutron separation energy. The consecutive beta decay gives rise to the creation of electrons and anti-neutrinos and to the consecutive emission of betadelayed gammas and neutrons. Structural effects are found in all of these processes.

\section{Conventional modeling}

Attempts for modeling the emission of prompt neutrons from fully accelerated fragments have a long tradition. The analytical Los-Alamos model [1] and later numerical computer codes (partly with the inclusion of gamma emission), e.g. [2-5], are in use to calculate the multiplicity distribution as well as the energy and angular distribution of the prompt

\footnotetext{
*e-mail: schmidt-erzhausen@t-online.de
} 
neutrons as a statistical evaporation process. This is done by imposing measured or estimated fission-fragment Apre-TKEpre distributions, measured fragment-mass dependent mean prompt-neutron multiplicities and estimated $\mathrm{Z}$ distributions for a given Apre as well as estimated fragment angular-momentum distributions, which define the starting points of the evaporation cascades of the individual fragments. Models for the calculation of these starting conditions in case of scarce or missing experimental data, for example with a semiempirical description [6], with microscopic calculations [6, 7] or with machine learning [8] are under development. Difficulties arise to obtain the necessary accuracy, to cover the multitude of the fission quantities, to assure the wide-spread correlations between them, and to make predictions for systems with scarce or no experimental information.

\section{Features of a complete model}

A complete model of light-particle emission in fission must consider the whole fission process, beginning from pre-equilibrium emission and evaporation of light particles before fission, the emission of mostly neutrons on the way to scission, eventually the production of scission neutrons, which is not finally confirmed, a model for the properties of the fission fragments, e.g. fragment yields, thermal excitation energy at scission and its division between the fragments, other contributions to the excitation energies of the final fragments, e.g. the release of deformation energy after scission, a model for the generation of fragment angular momentum and, as the last steps of prompt emission the evaporation of prompt neutrons and the emission of photons from the fragments, including the kinematical transformations from the fragment frame to the laboratory. Finally, the radioactive decay chains of the neutron-rich fragments must be considered.

The semi-empirical GEF model [9] fulfills most of these requirements. The flow diagram of GEF (Fig. 1) gives a graphical presentation of the described steps of the fission process and illustrates the complexity and the inherent correlations of the different fission quantities.

\section{Some structural effects in comparison with the GEF model}

This section is devoted to the description of some important basic ideas of the GEF model and to a comparison of specific results of the GEF code with experimental data, in view of light-particle production and gamma radiation in fission. For a comprehensive presentation of the GEF model, we refer to ref. [9].

\subsection{Variation of fission yields with universal fragment shells}

The regularities of fission channels as a function of the composition of the fissioning system and its excitation energy prove that the shell effects on the fission path evolve in a non-erratic way. The two-centre shell model provided the explanation that the shells slightly beyond the outer fission barrier are strongly determined by the fragment shells [10]. Therefore, only the macroscopic potential is characteristic for the fissioning system, while the shell effects are attributed to the fragments. This means that these shell effects are universal for all fissioning systems. The variation of the fission yields is caused by the superposition of the macroscopic and the microscopic contributions. This is illustrated in Fig. 2 on the example of the transition from predominantly symmetric to predominantly asymmetric fission around thorium.

In addition, observations [12] and theoretical considerations [13] suggest that the influence of proton shells is dominant. Thus, a proton shell at large deformation around $Z=$ 55 (acting on the heavy fragment) seems to be responsible for the asymmetric fission in the actinides. However, also weaker shells in the light fragment and shells in the neutron subsystems of the light and the heavy fragment should have some additional influence. 


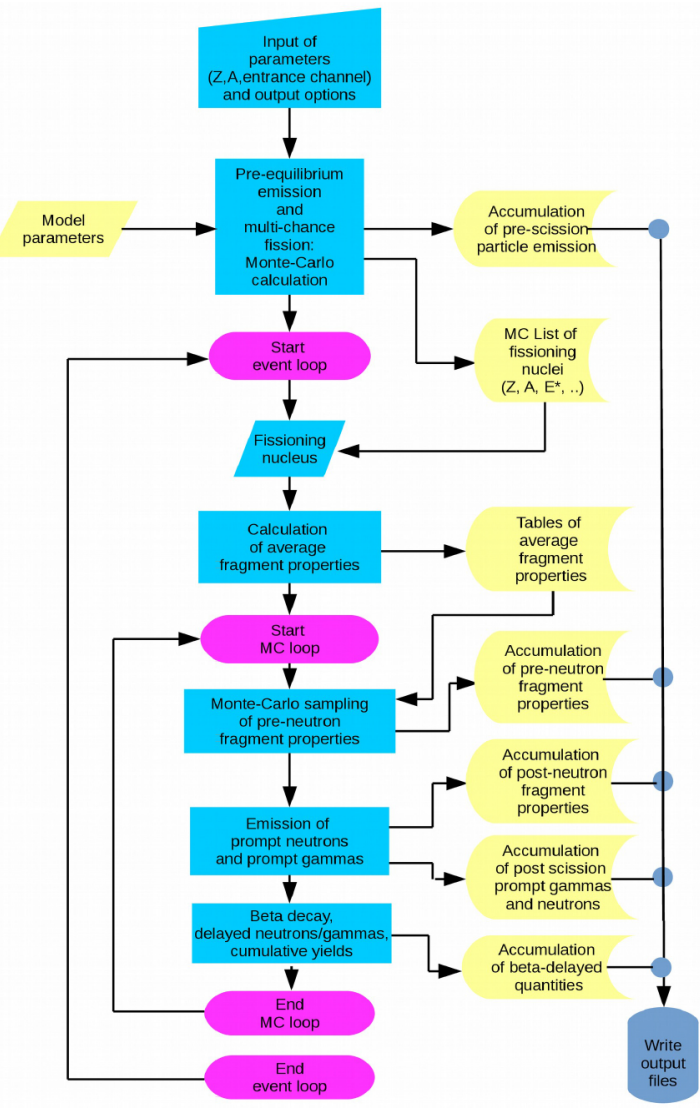

Figure 1. Flow diagram of the GEF code. (Figure modified from Ref. [9].)

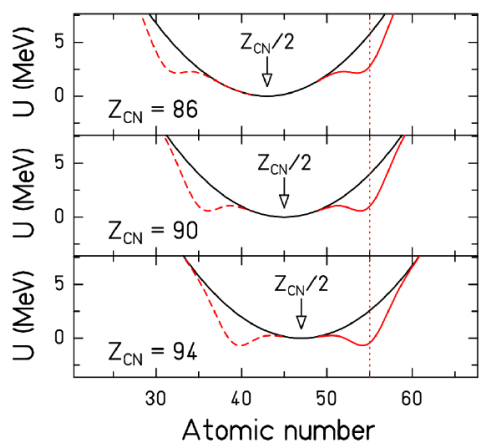

Figure 2. Schematic drawing of the superposition of the macroscopic potential (black) and a fragment shell at $Z=55$ (red) for different fissioning systems. (Figure from Ref. [9].)

\subsection{Shell-determined fragment deformation at scission}

It is a well known result of macroscopic-microscopic calculations (e.g. Ref. [11]) that shells at large deformation show a correlation between the particle number and the optimum deformation. This correlation can explain the saw-tooth behaviour of the mean prompt-neutron 


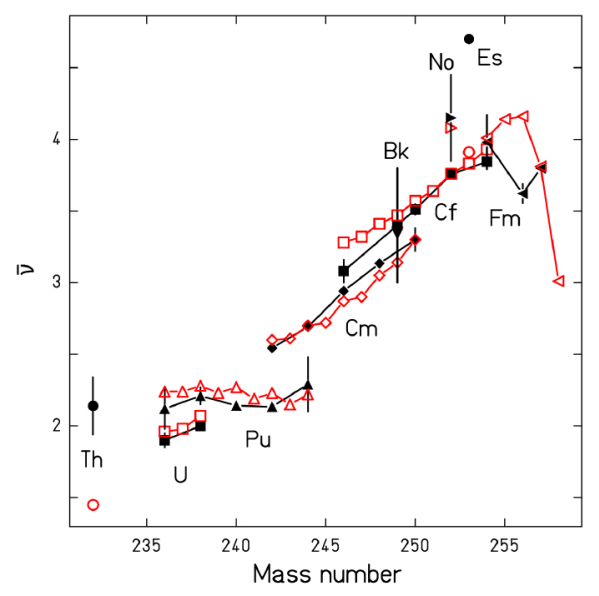

Figure 3. Measured prompt-neutron multiplicities in spontaneous fission (black symbols) in comparison with GEF calculations (red symbols). (Figure from Ref. [9].)

multipicity in the fission of actinides. Together with the dominance of proton shells mentioned above, the contribution to the excitation energy of the fission fragments from the fragment deformation at scission is expected to be determined by the number of protons in the fragment.

\subsection{Mean prompt-neutron multiplicities}

With the ideas from Sects. 4.1 and 4.2 and a suitable unique set of model parameters, GEF is able to reproduce the mean prompt-neutron multiplicities as a function of the composition of the fissioning system to a great extent. Fig. 3 shows a comparison for spontaneous fission, where the structural effects are especially strong. There is a general increase of the neutron multiplicity with increasing mass along isotopic chains. The rather constant value for the $\mathrm{Pu}$ isotopes is explained by a relatively strong yield of the $\mathrm{S} 1$ fission channel that increases with neutron excess of the fissioning system. This feature is attributed to a shell in the light fragment near $Z=38$, which is also assumed to be responsible for the double-humped fission of nuclei near ${ }^{180} \mathrm{Hg}$ [6]. The situation in the Fm isotopes is unclear, may be due to experimental uncertainties. Experimental verification of ${ }^{256} \mathrm{Fm}$ and additional data for other isotopes would be welcome.

\subsection{Thermal energy at scission}

Before scission, both nucleons and excitation energy can be exchanged between the nascent fragments through the neck. Commonly, the behaviour of such a di-nuclear system in fission or in binary heavy-ion reactions is assumed to be governed by statistical mechanics. e.g. by the tendency to acquire thermal equilibrium. In low-energy fission, the fissioning system is under the influence of pairing correlations, which means that the nuclear temperatures of the two nascent fragments do (almost) not depend on excitation energy. Due to the mass dependence of the nuclear temperature $\left(\mathrm{T} \sim \mathrm{A}^{-2 / 3}\right)$, it is expected that the thermal energy available before scission is absorbed by the heavy fragment, because it has the lower temperature. This hypothesis has been tested by an experiment, where the incident energy in neutron-induced fission of ${ }^{237} \mathrm{~Np}$ was increased from $0.8 \mathrm{MeV}$ to $5.55 \mathrm{MeV}$ [14]. 


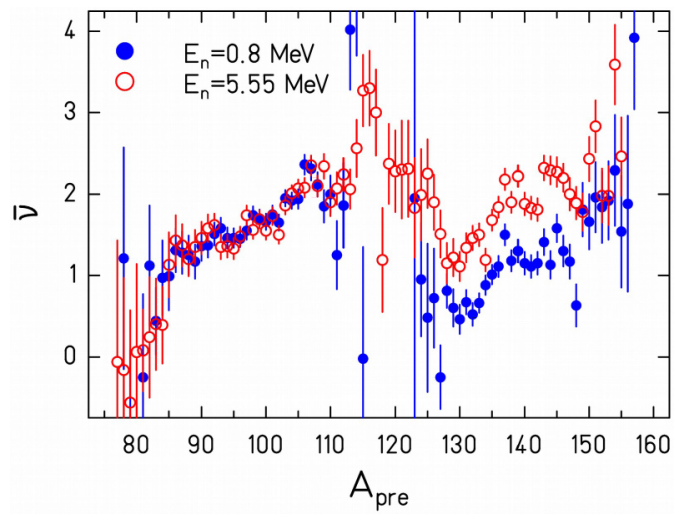

Figure 4. Mean multiplicity of prompt neutrons in the reaction ${ }^{237} \mathrm{~Np}(\mathrm{n}, \mathrm{f})$ with two different incident neutron energies as a function of fragment mass. Data from Ref. [14], figure from Ref. [9].

Indeed, the prompt-neutron multiplicity in the light fragment group did not change, while it increased all over the heavy-fragment range, see Fig. 4.

One could argue that the nuclei around the doubly-magic ${ }^{132} \mathrm{Sn}$ have strong shell effects, which increases the nuclear temperature. This could lead to an inversion of the energy-sorting process. However, in the relevant configuration at scission, this is only true for the fragments formed in the S1 fission channel. According to the GEF calculation shown in Fig. 5, the S1 fission channel has only a yield of $14.4 \%$, and the yield is only comparable to the one of the S2 channel around $\mathrm{A}=107$ and $\mathrm{A}=130$. There is indeed an indication for an incomplete energy sorting in this region in Fig. 4, but the statistical significance is low. Thus, an eventually inverse energy sorting of fragments of the $\mathrm{S} 1$ channel is not in conflict with the experiment.

\subsection{Prompt emission of gammas}

GEF includes a competition between fission, light-particle emission and gamma emission from the first minimum of the initial system and between the emission of neutrons and gammas from the fragments. The gamma strength for the statistical emission of E1 radiation is given by the GDR as proposed in Ref. [15].

The prompt emission of non-statistical prompt gammas is governed by the complex spectroscopic nuclear properties. In the GEF code, these are described by an updated VMI (variable moment-of-inertia model). This makes calculations possible for nuclei with no or incomplete experimental spectroscopic information. The comparison with measured state energies shows rather good agreement, see Fig. 6.

The transition from rotational to vibrational structure in the vicinity of closed shells is well reproduced, although there are deviations for nuclei very near to closed shells.

Figure 7 shows rather good agreement for the low-energy part of the prompt-gamma spectrum for ${ }^{239} \mathrm{Pu}\left(\mathrm{n}_{\mathrm{th}}, \mathrm{f}\right)$. Note the higher resolution in the calculation!

\subsection{Production of anti-neutrinos}

The understanding of the anti-neutrino production in fission and the theoretical calculation of the anti-neutrino energy spectra in different, also future, types of fission reactors rely on the application of the summation method, where the individual contributions from the different 


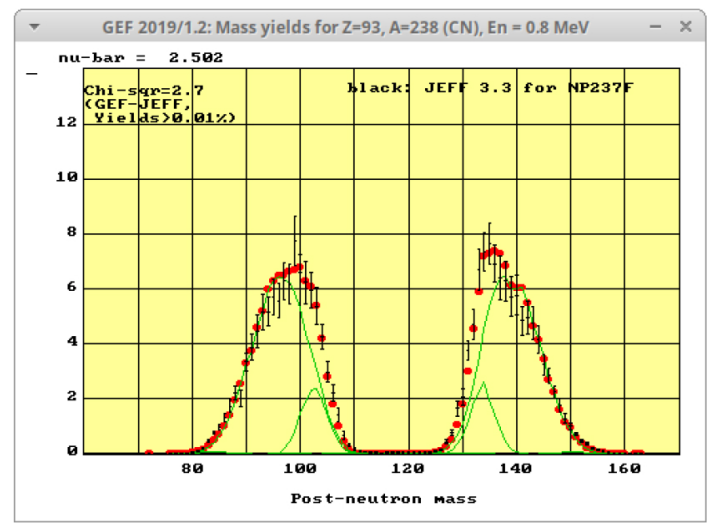

Figure 5. Mass dependent fission yields (after prompt-neutron emission) from GEF (red points) in comparison with JEFF 3.3 (black symbols with error bars). The green curves represent the contributions of the different fission channels. S2 is centered around $\mathrm{A}=96$ and $\mathrm{A}=137$, while $\mathrm{S} 1$ is centered around $\mathrm{A}=103$ and $\mathrm{A}=133$.

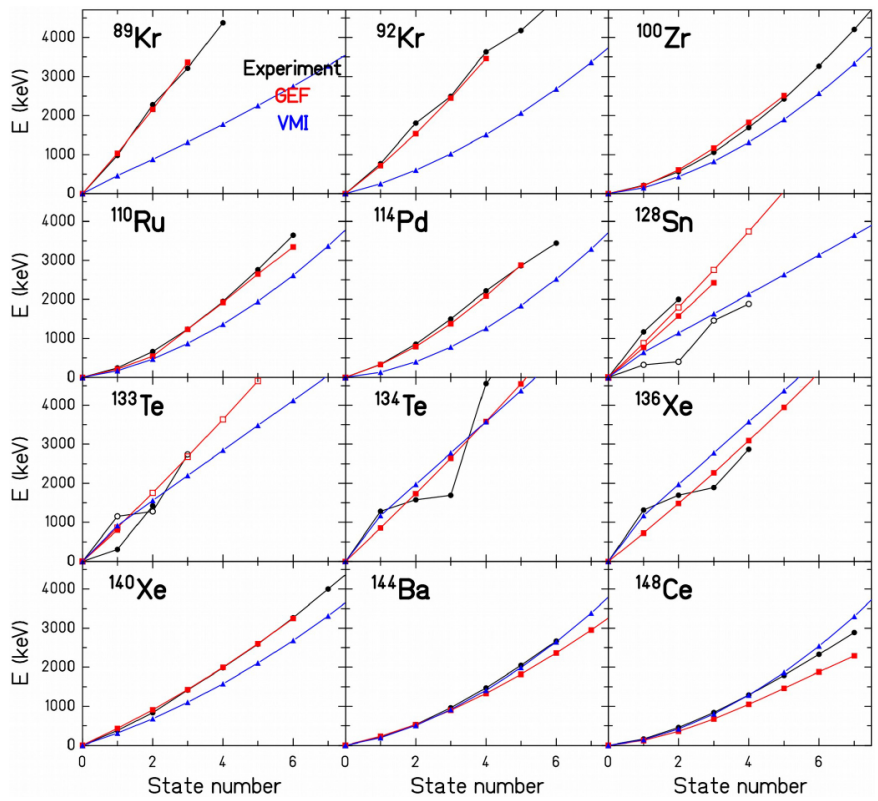

Figure 6. Experimental state energies (black dots) are compared with a rather recent VMI model [16] (blue) and the GEF model (red). Figure from [9].

radioactive nuclides that undergo a beta decay are estimated and summed up. Figure 8 shows the cumulative fission yields and the $Q$ values of the beta decays for the system ${ }^{235} \mathrm{U}\left(\mathrm{n}_{\mathrm{th}}, \mathrm{f}\right)$, calculated with GEF by use of the JEFF 3.1.1 decay tables. The cumulative yields indicate the magnitude of the contributions of the different nuclei to the anti-neutrino spectrum, while the $\mathrm{Q}$ values give a hint on the maximum energy of these contributions. Apparently, the highest energies are expected for the most neutron-rich odd- $Z$ light fission fragments. 


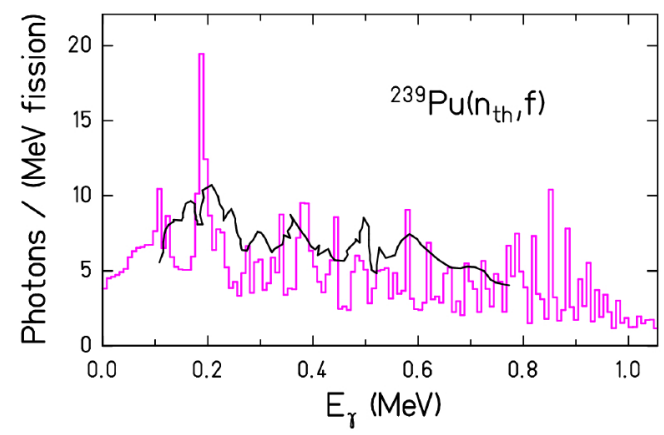

Figure 7. Lower part of the experimental prompt-gamma spectrum [17] (black line) of ${ }^{239} \mathrm{Pu}\left(\mathrm{n}_{\mathrm{th}}, \mathrm{f}\right)$ in comparison with the GEF result (pink histogram).

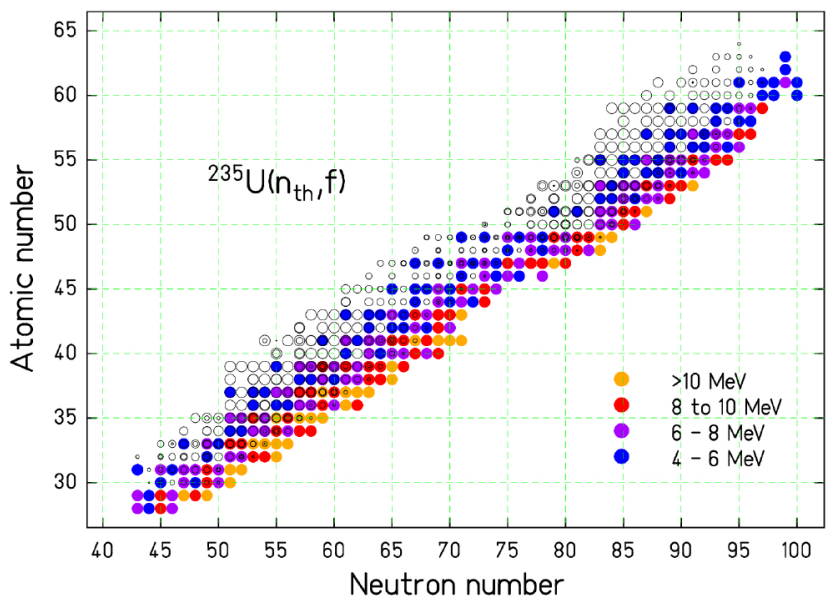

Figure 8. Cumulative yields (size of the black circles) and beta $Q$ values (coloured dots) for the thermalneutron-induced fission of ${ }^{235} \mathrm{U}$ from GEF. (Multiple black circles refer to the yields of isomers.)

Figure 9 shows the calculated anti-neutrino spectra from GEF for thermal-neutroninduced fission of different systems. The spectra show structures that are characteristic for the fissioning system and a tendency for the emission of high-energy neutrinos with increasing neutron excess. The accurate theoretical calculation of these spectra with their specific structures is a pre-requisite for an eventual remote monitoring of fission reactors.

\subsection{Emission of delayed neutrons}

The data on the evolution of delayed-neutron emission as a function of excitation energy are scarce. Nevertheless, there are clear indications for structural effects, see Fig. 10.

The GEF code reproduces the magnitude for the different systems rather well, while there is at least some qualitative similarities in the structures. The steep slope for even- $Z$ systems around $5 \mathrm{MeV}$ is well reproduced for ${ }^{238} \mathrm{U}(\mathrm{n}, \mathrm{f})$ and not so well for other systems. It is created by and very sensitive to the energy dependence of the pairing gap. Another structure near $15 \mathrm{MeV}$ is caused by multi-chance fission. 


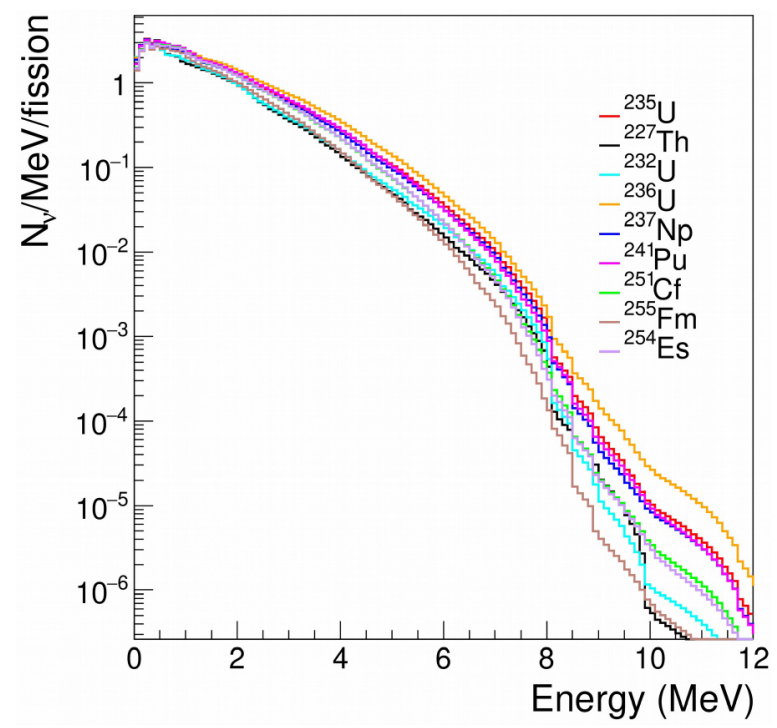

Figure 9. Calculated anti-neutrino spectra from GEF combined with the selection of decay data of [18] for different systems in logarithmic scale.
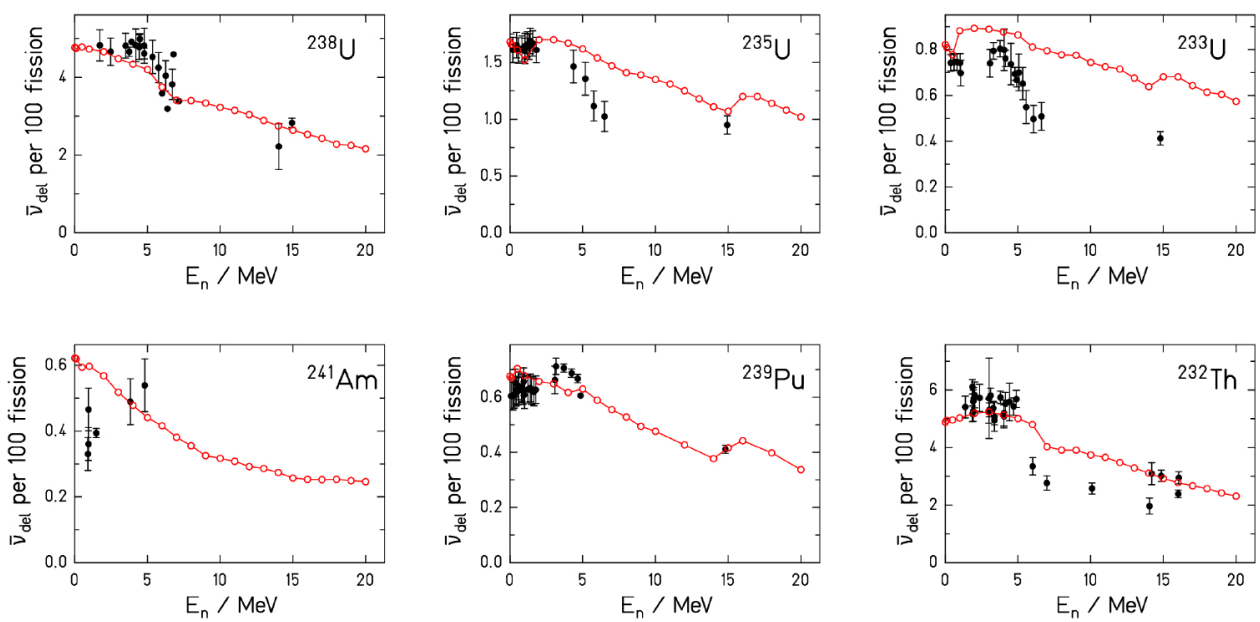

Figure 10. Energy dependence of the delayed neutron yield in neutron-induced fission of several systems. The data (black symbols) are compared with GEF results (red symbols). Figure from [6].

\section{Conclusion}

Manifold and complex structural features appear in light-particle production accompanying nuclear fission. They appear as local discontinuities, deviations from smooth trends or peak structures in different observables. Calculations that are able to reproduce these features and to make reasonable predictions in cases of scarce or missing experimental information must be based on a comprehensive theoretical model. In this contribution, the GEF model is given as an example and compared with experimental data for a number of selected observables. 
Due to the underlying concepts and laws of general validity, and the consistent coverage of practically all fission quantities, it provides reliable results over a large range of systems (in view of nuclear composition and excitation energy), including the structural effects, with a unique description, when the parameters are determined on the basis of a limited amount of experimental data.

\section{References}

[1] D.G. Madland, A.C. Kahler, Nucl. Phys. A 957 (2017) 289

[2] I. Stetcu, P. Talou, T. Kawano, M. Jandel, Phys. Rev. C 90 (2014) 024617

[3] P. Talou, T. Kawano, I. Stetcu, Nucl. Data Sheets 118 (2014) 195

[4] O. Litaize, O. Serot, L. Berge, Eur. Phys. J. A 51 (2015) 177

[5] R. Vogt, J. Randrup, Phys. Rev. C 96 (2017) 064620

[6] K.-H. Schmidt, B. Jurado, Rep. Progr. Phys. 81 (2018) 106301

[7] N. Schunck, L.M. Robledo, Rep. Prog. Phys. 79 (2016) 116301

[8] Zi-Ao Wang, Junchen Pei, Yue Liu, Yu Qiang, Phys. Rev. Lett. 123 (2019) 122501

[9] K.-H. Schmidt, B. Jurado, C. Amouroux, C. Schmitt, Nucl. Data Sheets 131 (2016) 107

[10] U. Mosel, H.W. Schmitt, Phys. Rev. C 4 (1971) 2185

[11] B.D. Wilkins, E.P. Steinberg, R.R. Chasman, Phys. Rev. C 14 (1976) 1832

[12] C. Böckstiegel et al., Nucl. Phys. A 802 (2008) 12

[13] G. Scamps, C. Simenel, Nature 564 (2018) 382

[14] A.A. Naqvi et al., Phys. Rev. C 34 (1986) 218

[15] A.R. Junghans et al., Phys. Lett. B 670 (2008) 200

[16] J.S. Batra, Raj K. Gupta, Phys. Rev. C 43 (1991) 1725

[17] A. Gatera et al., Phys. Rev. C 95 (2017) 064609

[18] M. Estienne et al., Phys. Rev. Lett. 123 (2019) 022502 